\title{
Radioanatomical Study of the Pedicle of the Superficial Circumflex Iliac Perforator Flap
}
${ }^{1}$ Fondazione Policlinico Universitario A. Gemelli IRCCS, Dipartimento Scienze Della Salute Della Donna E Del Bambino E Di Sanità Pubblica, Unità di Chirurgia Plastica, Roma, Italia
2 Fondazione Policlinico Universitario A. Gemelli IRCCS, Dipartimento Scienze Della Salute Della Donna E Del Bambino E Di Sanità Pubblica, Roma, Italia
3 Università Cattolica del Sacro Cuore, Istituto di Clinica Ostetrica e Ginecologica, Roma, Italia
${ }^{4}$ Università Cattolica del Sacro Cuore, Istituto di Clinica Chirurgica, Roma, Italia
${ }^{5}$ Fondazione Policlinico Universitario A. Gemelli IRCCS, Dipartimento Diagnostica per Immagini Radioterapia oncologica ed Ematologia, Roma, Italia

Stefano Gentileschi, MD, PhD ${ }^{1,4}$ Maria Servillo, MD ${ }^{4}$ Francesca De Bonis, MD

Roberta Albanese, $\mathrm{MD}^{4}$ Valentina Pino, $\mathrm{MD}^{4}$ Maria Lucia Mangialardi, MD ${ }^{4}$ lacopo Valente, $\mathrm{MD}^{5}$

Giorgia Garganese, MD ${ }^{2}$ Giovanni Scambia, MD ${ }^{2,3}$ Marzia Salgarello, MD ${ }^{1,4}$ Alessandro Cina, MD

\author{
Address for correspondence Stefano Gentileschi, MD, PhD, \\ Fondazione Policlinico Universitario A. Gemelli IRCCS, Dipartimento \\ Scienze Della Salute Della Donna E Del Bambino E Di Sanità Pubblica, \\ Largo Agostino Gemelli 1-00168, Roma, Italia \\ (e-mail: stefanogentileschi@gmail.com).
}

J Reconstr Microsurg 2019;35:669-676.

\begin{abstract}
\section{Keywords}

- superficial circumflex iliac perforator flap

- superficial circumflex iliac artery

- groin flap

- radioanatomical study

- groin dissection

- perforator flap

Background The superficial circumflex iliac perforator (SCIP) flap has many ideal features, such as fast dissection, possibility to harvest thin, pliable, wide skin island, and concealed donor site scar. In spite of these features, its use was limited because of the wide anatomical variation of the pedicle, which is relatively shorter and has a smaller caliber than other more popular perforator flaps. Several names were given to the branches and perforators in the literature, thus adding confusion to the understanding of its anatomy. Methods We performed a surgical and a radioanatomical study of the SCIP pedicles analyzing high-resolution contrast-enhanced computed tomography (CT) scan of 95 groins, with particular attention to the deep branch (DB) of the superficial circumflex iliac artery (SCIA). Twenty-three of these patients were also studied by detecting the surgical anatomy during SCIP flap harvest. We employed a system of coordinates based on the line between pubic tubercle (PT) and anterior superior iliac spine (ASIS) to describe the position of the perforator of the DB.

Results We found a $100 \%$ correlation between surgical and radiological findings. The length of the DB from the origin to the point in which its perforator pierced the sartorius fascia ranged from 1.6 to $6.5 \mathrm{~cm}$, mean $=3.62 \pm 0.92 \mathrm{~cm}$. The distance between the origin of the DB and the inguinal ligament ranged from 1.1 to $7.5 \mathrm{~cm}$, mean $=2.8 \pm 1 \mathrm{~cm}$. The perforator of the DB could be found in $91 \%$ of the cases within a box of $4 \mathrm{~cm} \times 3 \mathrm{~cm}$ drawn caudally to the line joining the PT with the ASIS. This vessel can show a vertical or horizontal course in the subcutaneous layer.

Conclusion Our findings confirm other previous studies and add new information about the position and the course of the perforator of the DB of the SCIA. Important features of the SCIP pedicles can be investigated by the color Doppler ultrasound and CT scan.
\end{abstract}

received

February 22, 2019

accepted after revision

May 28, 2019

published online

July 17, 2019
Copyright $\odot 2019$ by Thieme Medical Publishers, Inc., 333 Seventh Avenue, New York, NY 10001, USA. Tel: +1(212) 584-4662.
DOI https://doi.org/

10.1055/s-0039-1693144. ISSN $0743-684 X$. 
McGregor and Jackson described the first groin flap in $1972{ }^{1}$ and the following year its successful transfer as a free flap was reported by Daniel and Taylor. ${ }^{2}$ In the era of perforator flaps, Koshima et al described the evolution of the groin flap: the superficial circumflex iliac perforator (SCIP) flap. ${ }^{3}$

Compared to the groin flap, the SCIP flap has a longer pedicle $^{4}$ that allows using it also as a pedicled flap ${ }^{5-7}$ and can be harvested as super-thin flap, ${ }^{8-10}$ reducing the need for secondary procedures of debulking and allowing minor donor site morbidity, in particular decreasing the risk of lymphatic leaking. ${ }^{11}$ Other valuable features of this flap are concealed donor scar and good skin texture, with the possibility to raise big flaps, achieving primary closure, particularly in patients undergone to weight loss. ${ }^{12}$ Despite these ideal features, the SCIP flap has not gained a widespread popularity, mainly because its vascular pedicle is not considered always optimal for microvascular anastomosis due to its small caliber and wide anatomical variation. Furthermore, the descriptions of the position and nomenclature of the branches of the superficial circumflex iliac artery (SCIA) and their perforators are ambiguous in the literature; they have been classified by different authors either according to their depth or site of origin. They were named either "superficial and deep" ${ }^{13}$ or "medial and lateral," 14 respectively, that is, the medial perforator arises from the superficial branch, while the lateral perforator arises from the deep branch. Feng et al named the perforators of the branches of the SCIA "proximal perforators" (from the medial or "superficial" branch) and "distal perforators" (from the lateral or "deep" branch). ${ }^{15}$

We conducted a surgical and radioanatomical study to understand the position of the SCIP pedicles in more detail, and to further investigate their course in the subcutaneous layer, with a particular focus on the lateral perforator of the deep branch.

\section{Methods}

In the first part of the study, we prospectively analyzed 23 consecutive patients undergoing unilateral groin region reconstruction with pedicled SCIP flap. All patients were females who underwent vulvectomy for vulvar carcinoma or melanoma with an inguinal defect caused by lymphadenectomy. ${ }^{6,16}$ Inguinal defect was in 20 cases a wide subcutaneous defect following extended groin dissection with 3 of them including also an inguinal skin resection because of proximity of pathologic nodes, and in three cases was caused by the removal of a big lymphocele, developed after groin dissection. ${ }^{17}$ Age, height, weight, and body mass index (BMI) of patients were recorded. Every patient had undergone preoperative high-resolution contrast-enhanced total body computed tomography (CT) scan, with focus at the level of genitalia and groins for cancer staging. Patients underwent the color Doppler ultrasound (CDU) of the groins to investigate SCIP pedicles anatomy and perforators' flow and position (-Fig. 1).

\section{Computed Tomography Technique}

Computed tomography angiography (CTA) imaging was performed with patients' supine, arms lying downward. CTA was performed using 64-slice multidetector CT (LightSpeed VCT, GE Healthcare, Waukesha, WI). Slice thickness was $0.65 \mathrm{~mm}$, table travel per rotation $23 \mathrm{~mm}$ (gantry rotation time $=0.4$ seconds), field of view (FOV) $40 \mathrm{~cm}$ in order to match patient

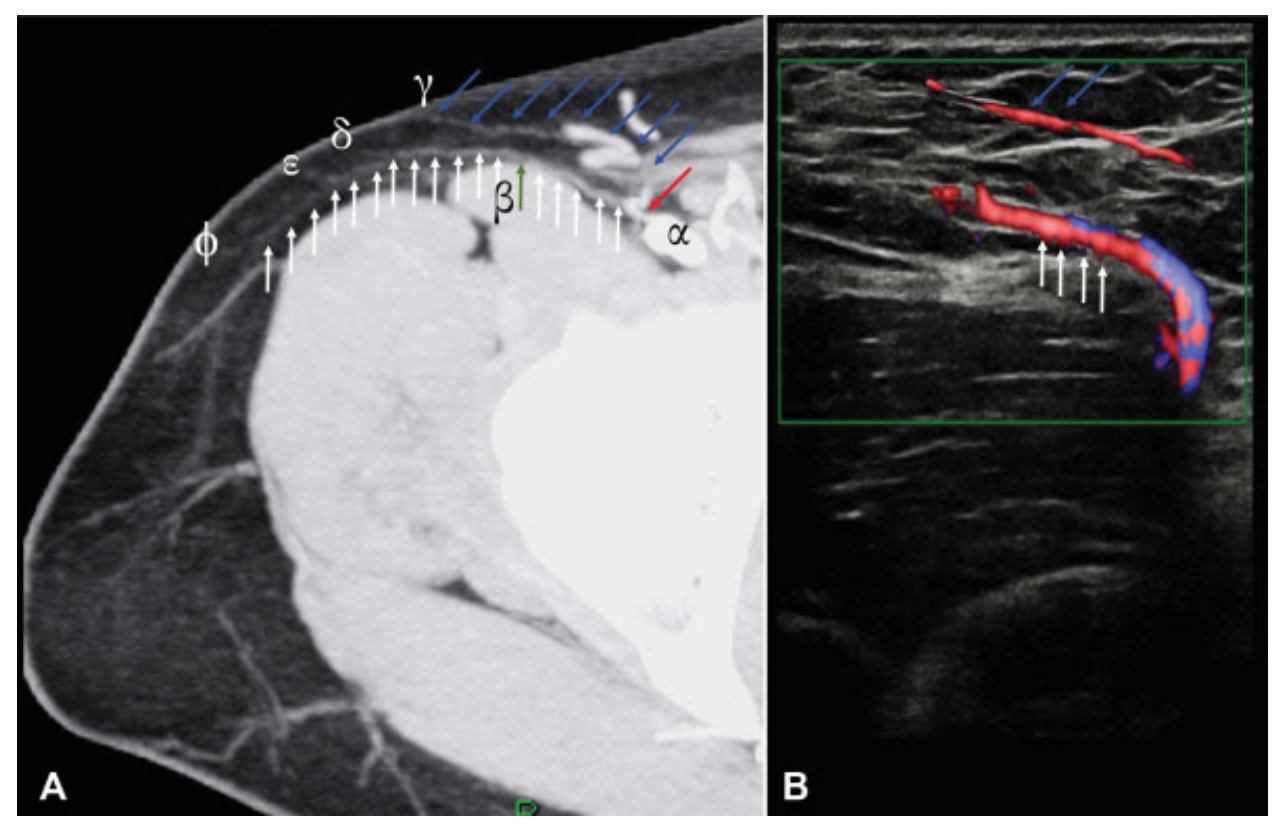

Fig. 1 (A) The picture shows the appearance of the SCIP pedicles in the CT scan. $\alpha$ indicates the femoral artery and the red arrow indicates the common trunk of the SCIA. The blue arrows indicate the superficial branch of the SCIA, supplying lymph nodes and going toward the dermis (point $\gamma$ ). The white arrows indicate the deep branch of the SCIA, entering under the sartorius $(\beta)$ fascia and giving off the perforator (green arrow) that pierces the fascia and courses into the subcutaneous layer of the flank, showing many vertical branches toward the dermis ( $\delta$, $\varepsilon$, $\phi$ ). (B) The picture shows the color Doppler ultrasound of the same groin. The blue arrows indicate the superficial branch of the SCIA, going toward the skin and the white arrows indicate the deep branch of the SCIA. CT, computed tomography; SCIA, superficial circumflex iliac artery; SCIP, superficial circumflex iliac perforator. 
width, matrix size $512 \times 512$ voxels. Tube voltage was $120 \mathrm{kVp}$, with Smart mAs (GE Healthcare) dose enabled (noise index set to 22). Acquisition range extended from $3 \mathrm{~cm}$ above the umbilicus to the upper thigh. Image acquisition was performed in a caudal-cranial direction, during the intravenous administration of $100 \mathrm{~mL}$ iodinated contrast medium (Iopamiro, Bracco Imaging, Milan, Italy) at $400 \mathrm{mg} \mathrm{i} / \mathrm{mL}$ concentration. Contrast was administered through a double-barrel injector, with an 18-G cannula placed into an antecubital vein, at $3.5 \mathrm{~mL} / \mathrm{s}$ flow rate, followed by $60 \mathrm{~mL}$ saline flush. Bolus tracking (Smart Prep, GE Healthcare, Waukesha, WI) was enabled, with the region of interest (ROI) placed on the proximal superficial femoral artery, in order to synchronize acquisition time with the arterial peak of enhancement in this vessel.

For CTA, data set acquired was postprocessed and analyzed on a dedicated workstation (Advantage Windows 4.1, GE Healthcare). Vessels object of this study were identified on axial subvolume maximum intensity projection (MIP) reconstructions and their calibers measured.

In the CT scans, we detected and recorded the following features of the SCIP pedicles: (1) the presence of the superficial and deep branches of the SCIA; (2) their caliber at the origin; (3) the presence of common or separate origin from the femoral artery; (4) the length of the common trunk, if present, or the distance between the two origins if separate; (5) the presence of the perforator of the deep branch; (6) the length of the deep branch from the origin to the point in which its perforator pierced the sartorius fascia; and (7) the distance between the origin of the deep branch and the inguinal ligament that calculated along the course of the femoral artery. The distance between the pubic tubercle (PT) and the anterior superior iliac spine (ASIS) and the position of the point in which the perforator of the deep branch pierced the sartorius fascia were calculated employing a virtual coordinate system, created on a volume rendered (VR) reconstruction of the abdominal and groins surface. We considered the $\mathrm{X}$ axis as the line between the PT and ASIS with the origin (X-axis value $=0 \mathrm{~cm}$ ) on the PT. The Yaxis was a second line, orthogonal to the $\mathrm{X}$ axis and passing from the PT, with its origin (Y-axis value $=0 \mathrm{~cm}$ ) on the PT. The point of intersection between the two lines was individuated as point " $\varnothing$ " (-Fig. 2). We also investigated the course of the perforators of the deep branch and of the superficial branch of the SCIA in the subcutaneous layer, recording if their direction was short and vertical toward the dermis, or long and horizontal, toward the flank.

During ablative surgery for vulvar cancer, a vessel sparing groin dissection ${ }^{18}$ was performed, saving, whenever possible, all the groin veins and arteries. The patients' lower limbs were abducted, flexed at the knee, and externally rotated. Inguinal lymphadenectomy was performed with a curvilinear incision below the inguinal ligament. The skin flaps were elevated following the thigh and abdominal superficial fascia. ${ }^{19,20}$

Special precautions were taken to avoid injuring the vascular pedicles of the inguinal region. As first step, the greater saphenous vein (GSV) was identified, isolated in the tract between the inferior angle of the femoral triangle and the saphenous

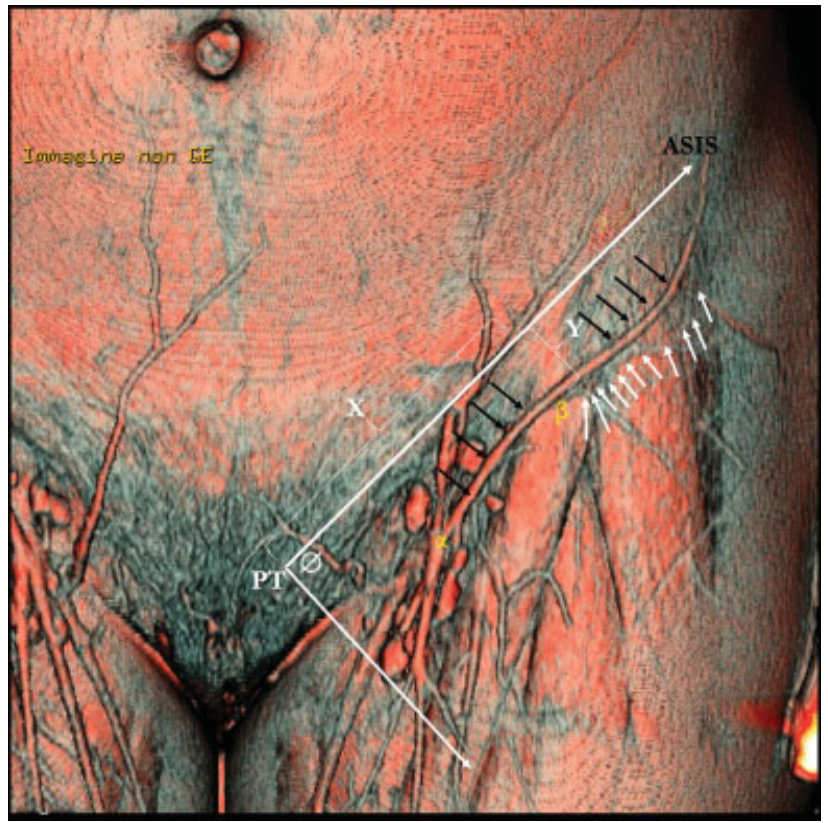

Fig. 2 The picture shows the virtual coordinates system, created on a volume-rendered reconstruction of the abdominal and groin surface, to locate the position of the perforator of the deep branch of the SCIA. $\alpha$ indicates the origin of the superficial branch of the SCIA, $\beta$ indicates the point at which the deep branch of the SCIA pierces the sartorius fascia. Black arrows indicate the course of the superficial branch of the SCIA. White arrows indicate the course of the perforator of the deep branch of SCIA in the subcutaneous layer. To locate the coordinates of point $\beta$, a line was drawn joining the PT with the ASIS. Then a second line, vertical to the first, was drawn, passing from the PT. The intersection of the two lines was considered the origin of the two axes and, therefore, value 0 . The $X$ - and $\mathrm{Y}$-axis values were calculated as distances in centimeters. PT, pubic tubercle; SCIA, superficial circumflex iliac artery.

crosse, and preserved. While dissecting the fibrofatty tissue surrounding the GSV toward the saphenous crosse, the origins of superficial inferior epigastric vein (SIEV), superficial circumflex iliac vein (SCIV), and internal pudendal vein were identified. These vessels were dissected from the surrounding fatty tissue and preserved. After the preservation of these veins, the superficial inferior epigastric artery (SIEA) and SCIA were identified and preserved. After vessels preservation, the superficial and deep inguinal lymph nodes were removed, cleaning the femoral triangle content, as previously described by Daseler et al. ${ }^{21}$

This technique differs from standard radical inguinal lymphadenectomy because the first part of surgery is guided by vessels isolation and preservation before fat and lymph nodes removal.

In particular, SCIA, SCIV, SIEA, SIEV, GSV, accessory saphenous vein, and the perforator of the deep branch of the SCIA were spared in all patients. Whenever possible, pudendal artery and vein were also spared. At the end of lymphadenectomy, we investigated the SCIP pedicles recording the same measures and features detected in the preoperative CT scan and CDU. The degree of matching between the CT, CDU, and surgical findings was also recorded at this moment. The SCIP flap was then harvested on the dissected pedicles and transposed to the groin region, filling the defect. 
We noticed a $100 \%$ degree of matching between CT, CDU and surgical findings in the 23 patients. The only difference was that when these vessels had a long horizontal subcutaneous course, the CDU was not able to follow them clearly beyond the ASIS, while CT scan could. This fact was not considered relevant because it is already known that CTA has a high-resolution ratio and can detect vessels with a caliber greater than $0.3 \mathrm{~mm},{ }^{22}$ whereas CDU is capable of detecting vessels with a caliber greater than $0.5 \mathrm{~mm} .{ }^{23}$

Therefore, we went on with the second part of the study in which we retrospectively reviewed high-resolution contrastenhanced CT scans of 72 inguinal regions, executed for preoperative evaluation of 36 female patients undergoing breast reconstruction with DIEP flap. Exclusion criteria were pathologic inguinal nodes, vascular pathologies, and previous surgery on the groins. The same features of the SCIP flap pedicle, described in the first part of the study, were investigated in the second part. The $\mathrm{CT}$ technique and the analysis of the images were the same as the first part of the study. Considering the complete concordance in these first 23 patients between preoperative $\mathrm{CT}$ and $\mathrm{CDU}$, imaging and surgical findings we added, the surgical and radioanatomical data of the first 23 , and following 72 groins, analyzing the results in a total of 95 inguinal regions.

\section{Results}

The age of the 59 patients ranged from 41 to 85 years, mean $=55.8 \pm 9.7$. BMI was ranged from 20 to $31 \mathrm{~kg} / \mathrm{m}^{2}$, mean $=24.7 \pm 2.78$. Among the 23 patients operated, in 8 cases the flap was harvested on the superficial branch of the SCIA, in 12 cases on the deep branch, and in 3 cases we included both.

In every patient, the superficial branch gave one or two perforators, while the medial branch gave one to three perforators. The dimension of the flap ranged from 32 to $220 \mathrm{~cm}^{2}$, (mean $\left.=111 \mathrm{~cm}^{2}\right)$. The whole flap was used in the three cases that ended up with a post excision skin defect, while in 20 cases with no skin defect; the flap was de-epithelialized and buried to fill the subcutaneous dead space and cover the blood vessels, leaving only a small skin island to monitor the flap. Marginal bleeding was excellent in all the 23 flaps. We observed two cases of dehiscence of the donor site scar, but no case of complication of the flap. Vulvar defect was repaired primarily except in three patients who needed a bilateral $\mathrm{V}-\mathrm{Y}$ fasciocutaneous advancement flap.

On the total of the 95 groins studied, in 86 (90.5\%) both the superficial and the deep branches were present, in $5(5.2 \%)$ the superficial branch was absent, while in $4(4.2 \%)$ the deep branch was absent. In every groin, where the deep branch was present, its perforator was detectable as well. The diameter of the superficial branch at its origin ranged from 1 to $2 \mathrm{~mm}$, mean $=1.55 \pm 0.25 \mathrm{~mm}$. The diameter of the deep branch at its origin ranged from 0.7 to $1.8 \mathrm{~mm}$, mean $=1.33 \pm 0.26 \mathrm{~mm}$. In 67 of the 86 groins, where both the superficial and the deep branches were present, the superficial branch was bigger than the deep branch $70.5 \%$ on the total of the 95 groins), whereas in 15 (15.7\% on the total of the 95 groins) it was smaller. In four groins ( $4.2 \%$ on the total of the 93 groins), they showed the same size. In 74 groins $(77.8 \%$ on the total of the 95 groins), the origin of the deep and superficial branch from the femoral artery was common, while in 12 (12.6\% on the total of the 95 groins), they showed separate origins. The length of the common trunk ranged from 2 to $38 \mathrm{~mm}$, mean $=8.2 \pm 6.46 \mathrm{~mm}$. In the 12 cases of separate origins of the superficial and deep branches, the distance between them ranged from 3 to $27 \mathrm{~mm}$, mean $=12.6 \pm 8 \mathrm{~mm}$, and in seven groins the superficial branch arose more proximally than the deep branch, while in five groins it was the opposite. The length of the deep branch from the origin to the point in which its perforator pierced the fascia of the sartorius muscle ranged from 1.6 to $6.5 \mathrm{~cm}$, mean $=3.62 \pm 0.92 \mathrm{~cm}$. In every groin examined the deep branch arose from the anterolateral aspect of the femoral artery, except one, in which it emerged from the profunda femoris as an isolated vessel. The distance between the origin of the deep branch and the inguinal ligament, calculated following the course of the femoral artery, ranged from 1.1 to $7.5 \mathrm{~cm}$, mean $=2.8 \pm 1 \mathrm{~cm}$.

Creating the virtual coordinates system to measure the point in which the perforator of the deep branch pierced the sartorius fascia we observed that distance between the PT and the ASIS ranged from 12 to $16.5 \mathrm{~cm}$, mean $=13.7 \pm 0.8 \mathrm{~cm}$. Considering this line as the $\mathrm{X}$ axis, with the origin (X-axis value $=0 \mathrm{~cm}$ ) on the PT and the $\mathrm{Y}$ axis as a second line, orthogonal to the $\mathrm{X}$ axis and passing from the PT, with its origin (Y-axis value $=0 \mathrm{~cm}$ ) on the PT, we found that the $\mathrm{X}$-axis value of the position of the point in which the perforator of the deep branch of the SCIA pierced the sartorius fascia ranged from 8.4 to $13 \mathrm{~cm}$, mean $=10.7 \pm 1.03 \mathrm{~cm}$. The $Y$-axis value ranged from 1 to $4 \mathrm{~cm}$, mean $=2.4 \pm 0.7 \mathrm{~cm}$.

The course of both the superficial branch and the perforator of the deep branch showed two different possible patterns that we called patterns $A$ and $B$. In pattern $A$, the vessel after its origin quickly went toward the dermis with a short-vertical subcutaneous tract. In pattern $\mathrm{B}$, the course was more horizontal, with a progressive going up toward the skin of the flank and a very long subcutaneous tract that followed the axis between the pubic tubercle and the anterior superior iliac spine. Among the 86 groins in which we could detect the presence of both the superficial and the deep branch with its perforator, in 39 of them (41\% on the total of the 95 groins), the perforator of the deep branch showed, in its subcutaneous tract, a pattern $\mathrm{A}$, and in 47 ( $49.4 \%$ on the total of the 95 groins), a pattern B. The superficial branch always showed the opposite pattern respect to the perforator of the deep branch (-Fig. 3 ).

The point in which the perforator of the deep branch pierced the sartorius fascia could be found in $91 \%$ of the cases within a rectangular area of $4 \mathrm{~cm} \times 3 \mathrm{~cm}$. This rectangular area can be drawn with the upper side parallel to the line joining the PT with the ASIS (-Fig. 4).

\section{Discussion}

Koshima et al, who first reported the SCIP flap, described the possibility to harvest it on the perforator coming from either 


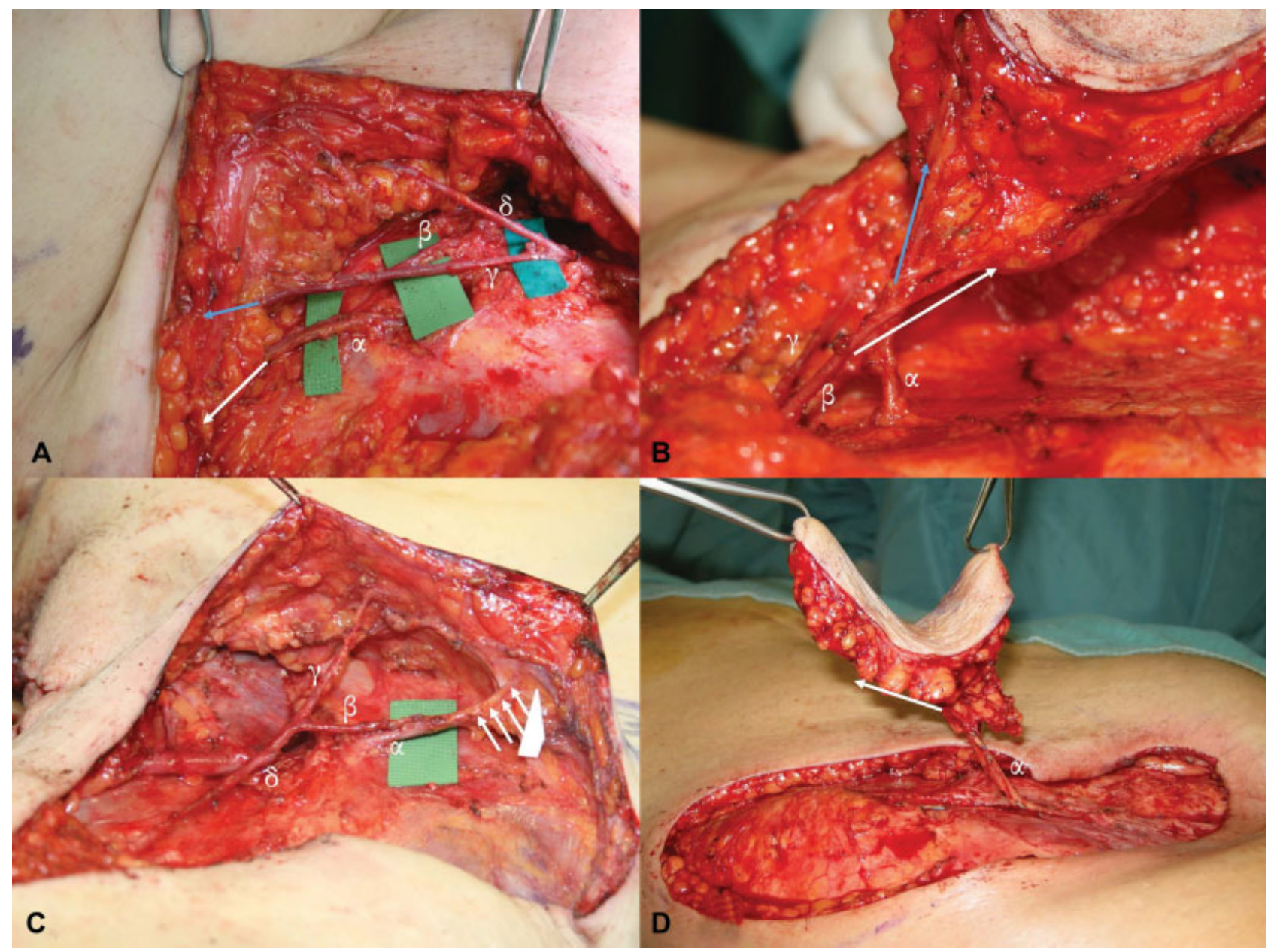

Fig. 3 Different patterns of SCIP pedicles. (A) Intraoperative view of the right groin represented in CT and CDU of the picture 1. $\alpha$ indicates the perforator of the deep branch of the SCIA, with his venae comitantes, and the white arrow indicates its horizontal direction in the subcutaneous layer (pattern B). $\beta$ indicates the superficial branch of the SCIA, joining with the SCIV ( $\mathrm{Y}$ ), and the blue arrow indicates its vertical direction going quickly toward the dermis (pattern A). $\delta$ indicates the superficial inferior epigastric vein (SIEV). (B) Intraoperative view of a left groin showing the two SCIP pedicles with opposite patterns respect to picture (A). The perforator of the deep branch of the SCIA ( $\alpha$ ) has a pattern A, going quickly toward the dermis (its direction is indicated by blue arrow), while the superficial branch ( $\beta$ ) shows a pattern B, following an horizontal course (indicated by the white arrow) in the subcutaneous layer. $\gamma$ indicates the SIEV. (C) In this left groin only the superficial branch of the SCIA is present $(\alpha)$. It joins with the SCIV $(\beta)$ and goes quickly toward the superficial dermal layer (white arrows), following a pattern A course. The SCIV $(\beta)$ has a common origin with the SIEV (Y) from the saphenous crosse $(\delta)$. (D) This picture shows a right groin in which only the perforator of the deep branch is present $(\alpha)$. It follows a horizontal course (pattern B), indicated by the white arrow. CDU, color Doppler ultrasound; CT, computed tomography; SCIA, superficial circumflex iliac artery; SCIP, superficial circumflex iliac perforator; SIEV, superficial inferior epigastric vein.

the superficial or the deep branch of the SCIA. ${ }^{3}$ In the description and in the drawings presented in his article, he reported the classic anatomy of the SCIA that arises from the femoral artery about $2.5 \mathrm{~cm}$ beneath the inguinal ligament and after a short course divides into two branches:

- The deep branch that enters under the sartorius fascia and, after giving off some muscular branches, ends with one main perforator that pierces the sartorius fascia at the lateral border of the muscle, and courses superolaterally in the subcutaneous layer of the flank, following the axis drawn between the PT and the ASIS.

- The superficial branch, that enters the subcutaneous fat of the groin region, supplying lymph nodes, going toward the ASIS and giving off some vertical branches toward the skin (that he called perforators of the superficial branch).
The deep branch ensures a longer pedicle to the flap, and is a true perforator, because after coursing under the sartorius fascia it pierces this fascia to go in the subcutaneous layer and nourish the overlying fat and skin. ${ }^{5}$ The superficial branch of the SCIA is described by some authors in the same way as a perforator, ${ }^{14,24}$ considering that the true origin of this vessel from the femoral artery is under the deep fascia, even if its course is almost completely in the subcutaneous layer, and generally this vessel is considered more a direct cutaneous vessel, rather than a true perforator. On the other side, Koshima et al observed that the superficial branch of the SCIA has generally a horizontal course in the subcutaneous layer and gives off some vertical branches that go toward the dermis. He called these small branches "perforators of the superficial branch of the SCIA" and showed that these little vessels allow to harvest very thin flaps. 

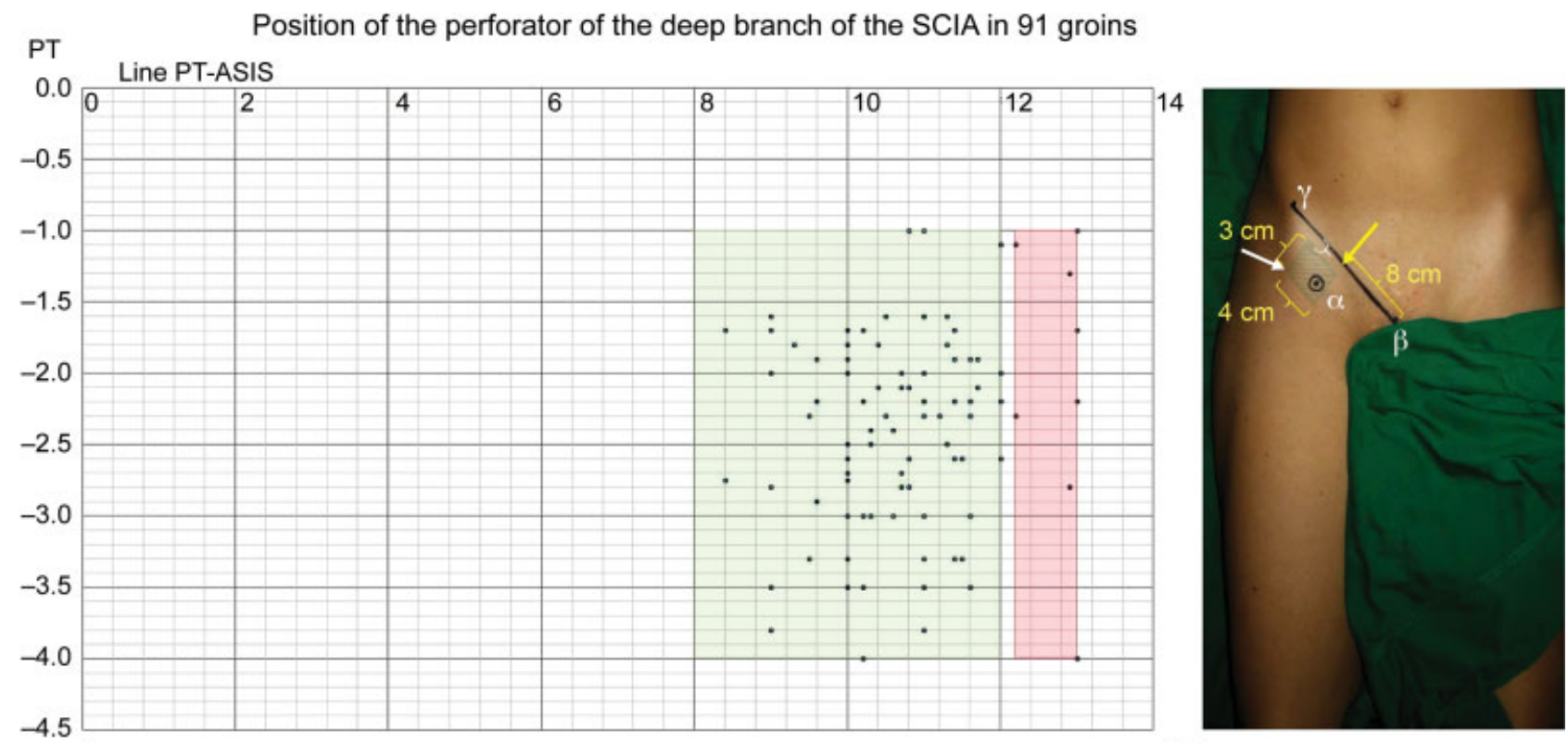

A

B

Fig. 4 (A) This plot shows the position of the perforator of the deep branch of the SCIA. The origin of the axis is the PT, and the X axis follows the line between the PT and the ASIS. Blue spots indicate the position of the perforator of the deep branch in every groin. Distances along the axis are indicated in $\mathrm{cm}$. Ninety-one percent of the perforators could be found in the green box measuring $4 \mathrm{~cm} \times 3 \mathrm{~cm}$. In the remaining $9 \%$ of the groins the perforator pierced the sartorius fascia in the area indicated in red. (B) The rectangular area (marked in green and indicated by the white arrow) where the perforator of the deep branch of the SCIA (black spot indicated by $\alpha$ ) could be found in $91 \%$ of the cases. It could be drawn with the upper side, measuring $4 \mathrm{~cm}$ parallel to the line joining the PT ( $\beta$ ) with the ASIS ( $\gamma), 1 \mathrm{~cm}$ inferior and lateral to this line (white bracket). The beginning of the upper side of the box is $8 \mathrm{~cm}$ lateral to the PT (yellow arrow), following the PT-ASIS line. The short sides are vertical to the first and measure $3 \mathrm{~cm}$. ASIS, anterior superior iliac spine; PT, pubic tubercle; SCIA, superficial circumflex iliac artery.

Feng et al reported that the superficial branch may give one or two perforators, while the medial branch may give one to three perforators and our results confirm the findings. ${ }^{15}$

Suh et al described these two vessels employing different terms ${ }^{14}$; the perforator of the deep branch of the SCIA is reported as the "lateral perforator"; the small vertical vessel from the superficial branch of the SCIA is called the "medial perforator." The findings of our study agree with those of Suh et al, showing that the superficial branch of the SCIA (or medial perforator) does not always take a horizontal course. In about $50 \%$ of patients, it has a vertical course and quickly enters the hypodermic and dermic layer. In about $40 \%$ of patients, it follows a horizontal course in the subcutaneous layer, giving off the small vertical branches previously described by Koshima et al. In addition to the findings of Suh et al, our study demonstrates that also the deep branch can show two different courses in the subcutaneous layer and in all the groins studied this course resulted opposite to the one of the superficial branch. The two possible courses can be vertical toward the dermis, penetrating soon the hypodermic and dermic layer near the ASIS (pattern A), or horizontal in the subcutaneous layer, toward the mid axillary line, parallel to the line joining the PT with the ASIS (pattern B).

We believe that even if we are living in the age of free style flaps, the preoperative knowledge of these anatomical details can be very important for a safer and faster flap planning and harvesting. In fact, given the choice between two possible pedicles, even if we assume that knowing before surgery, the origin and the position of the perforators is not so impor- tant, we believe that information about their course in the subcutaneous fat is a key factor in the flap planning. This is particularly true if a big flap is needed because the procedure is safer if we can rely on a pedicle with a long subcutaneous course (pattern $B$ ), rather than a short vertical course toward the dermis (pattern A). This information can help to foresee the length of the pedicle too. Unlike the study of Suh et al, ${ }^{14}$ we mainly focused the anatomy of the deep branch because our study begun with a clinical series of pedicled flaps employed for groin dissection defects after vulvar cancer surgery. Whenever possible, in these patients it is better to employ the deep branch that, despite the smaller vessels size, provides a longer pedicle, ensuring that the flap easily reach the inferior end of the femoral triangle. Another important advantage in choosing the deep branch, particularly when performing a free transfer of SCIP flap to reconstruct bony defects, is the possibility to harvest chimeric flap. ${ }^{25-27}$

Recently, Yoshimatsu et al have deepened the surgical anatomy of the SCIA with special focus on its contribution to the vascularization of the iliac bone and sartorius muscle studied by anatomical dissection, CT, and indocyanine green angiography of 10 fresh cadavers. ${ }^{28}$ These authors employed a coordinate system very similar to our system to describe the position of the perforator of the deep branch of the SCIA and showed that it is possible to harvest the cephalad portion of the sartorius muscle and the superficial portion of the iliac bone employing this vessel as a pedicle.

When the deep branch is employed as a pedicle, its anatomical relationship with the lateral femoral cutaneous 
nerve (LFCN) must be investigated. Usually, the deep branch runs beneath the LFCN. In a free SCIP transfer, the pedicle can be pulled under the LFCN after cut. On the contrary, in a pedicled transfer, the nerve must be cut and repaired. In our series, we repaired the nerve by direct coaptation. Postoperative sensory function recovery was not investigated in these patients; anyway, the possibility of a sensory deficit must be discussed with the patient before surgery. ${ }^{29}$

When performing a free transfer, to overcome the problem of short pedicle without giving up the advantage of the better size of the superficial branch, Yoshimatsu et al described a system to elongate a short-superficial pedicle by including the deep branch and using a retrograde flow. ${ }^{30}$ Dealing with the size of these vessels, we found that when both the superficial and the deep branches are present, usually one of the two is dominant on the other, and in most of the cases the bigger vessel is the superficial branch that confirms Koshima et al's description.

We believe that another important result of our study is the presence of the perforator of the deep branch within a rectangular area with defined anatomical landmarks (-Fig. 4) in $91 \%$ of the cases. This information can help to fasten the CDU study of patients and the flap planning.

One of the major drawbacks of this study is that the data are from only female patients. This may affect the average length and diameters of the SCIP pedicles vessels. On the other hand, vessels anatomical relationships, origins, and course in the subcutaneous tissue should not be affected by gender.

\section{Conclusion}

SCIP flap should be considered a workhorse flap, thanks to some ideal features. A great deal of preoperative information about vascular pedicles can be obtained by CDU or by contrast-enhanced high-resolution CT scan. In our opinion, preoperative CDU is useful before surgery in all the cases. CT scan can be useful in some cases, when a big flap is required, to obtain more precise information about the pattern of the vessels course in the subcutaneous layer. Whenever planning a pedicled flap, according to its size, the perforator of the deep branch should be chosen because it offers a longer pedicle. The possibility to foresee the position of the perforator of the deep branch of the SCIA can help the preoperative CDU study and the planning the flap.

Funding
None declared.

Conflict of Interest

None declared.

\section{References}

1 McGregor IA, Jackson IT. The groin flap. Br J Plast Surg 1972;25 (01):3-16

2 Daniel RK, Taylor GI. Distant transfer of an island flap by microvascular anastomoses. A clinical technique. Plast Reconstr Surg 1973; 52(02):111-117
3 Koshima I, Nanba Y, Tsutsui T, et al. Superficial circumflex iliac artery perforator flap for reconstruction of limb defects. Plast Reconstr Surg 2004;113(01):233-240

4 Iida T, Mihara M, Yoshimatsu H, Narushima M, Koshima I. Versatility of the superficial circumflex iliac artery perforator flap in head and neck reconstruction. Ann Plast Surg 2014;72(03):332-336

5 Gentileschi S, Servillo M, Garganese G, et al. The lymphatic superficial circumflex iliac vessels deep branch perforator flap: A new preventive approach to lower limb lymphedema after groin dissection-preliminary evidence. Microsurgery 2017;37(06):564-573

6 Gentileschi S, Servillo M, Garganese G, et al. Surgical therapy of vulvar cancer: how to choose the correct reconstruction? J Gynecol Oncol 2016;27(06):e60

7 Gentileschi S, Servillo M, Garganese G, Simona F, Scambia G, Salgarello M. Versatility of pedicled anterolateral thigh flap in gynecologic reconstruction after vulvar cancer extirpative surgery. Microsurgery 2017;37(06):516-524

8 Hong JP, Choi DH, Suh H, et al. A new plane of elevation: the superficial fascial plane for perforator flap elevation. J Reconstr Microsurg 2014;30(07):491-496

9 Iida T, Mihara M, Yoshimatsu H, Narushima M, Koshima I. Reconstruction of the external auditory canal using a super-thin superficial circumflex iliac perforator flap after tumour resection. J Plast Reconstr Aesthet Surg 2013;66(03):430-433

$10 \mathrm{Kim} \mathrm{JH}, \mathrm{Kim} \mathrm{KN}$, Yoon CS. Reconstruction of moderate-sized distal limb defects using a superthin superficial circumflex iliac artery perforator flap. J Reconstr Microsurg 2015;31(09):631-635

11 Choi DH, Goh T, Cho JY, Hong JP. Thin superficial circumflex iliac artery perforator flap and supermicrosurgery technique for face reconstruction. J Craniofac Surg 2014;25(06):2130-2133

12 Bracaglia R, Tambasco D, D'Ettorre M, Gentileschi S. "Inverted-Y”: a modified vest-over-pants abdominoplasty pattern following bariatric surgery. Aesthetic Plast Surg 2012;36(05):1179-1185

13 Sinna R, Hajji H, Qassemyar Q, Perignon D, Benhaim T, Havet E. Anatomical background of the perforator flap based on the deep branch of the superficial circumflex iliac artery (SCIP Flap): a cadaveric study. Eplasty 2010;10:e11

14 Suh HS, Jeong HH, Choi DH, Hong JP. Study of the medial superficial perforator of the superficial circumflex iliac artery perforator flap using computed tomographic angiography and surgical anatomy in 142 patients. Plast Reconstr Surg 2017;139(03): 738-748

15 Feng S, Xi W, Zhang Z, et al. A reappraisal of the surgical planning of the superficial circumflex iliac artery perforator flap. J Plast Reconstr Aesthet Surg 2017;70(04):469-477

16 Santonocito C, Concolino P, Lavieri MM, et al. Comparison between three molecular methods for detection of blood melanoma tyrosinase mRNA. Correlation with melanoma stages and S100B, LDH, NSE biochemical markers. Clin Chim Acta 2005;362(1-2):85-93

17 Gentileschi S, Servillo M, Salgarello M. Supramicrosurgical lymphaticvenous anastomosis for postsurgical subcutaneous lymphocele treatment. Microsurgery 2015;35(07):565-568

18 Garganese G, Collarino A, Fragomeni SM, et al. Groin sentinel node biopsy and ${ }^{18} \mathrm{~F}$-FDG PET/CT-supported preoperative lymph node assessment in CNO patients with vulvar cancer currently unfit for minimally invasive inguinal surgery: The GroSNaPET study. Eur J Surg Oncol 2017;43(09):1776-1783

19 Bracaglia R, Tambasco D, Gentileschi S, D'Ettorre M. Abdominoplasty in patient with penile implant. Aesthetic Plast Surg 2011; 35(05):933-935

20 Bracaglia R, D’Ettorre M, Gentileschi S, Tambasco D. “Vest over pants" abdominoplasty in post-bariatric patients. Aesthetic Plast Surg 2012;36(01):23-27

21 Daseler EH, Anson BJ, Reimann AF. Radical excision of the inguinal and iliac lymph glands; a study based upon 450 anatomical dissections and upon supportive clinical observations. Surg Gynecol Obstet 1948;87(06):679-694 


\section{Radioanatomical Study of the Pedicle of the SCIP Flap Gentileschi et al.}

22 Rozen WM, Phillips TJ, Ashton MW, Stella DL, Gibson RN, Taylor GI. Preoperative imaging for DIEA perforator flaps: a comparative study of computed tomographic angiography and Doppler ultrasound. Plast Reconstr Surg 2008;121(01):9-16

$23 \mathrm{Su}$ W, Lu L, Lazzeri D, et al. Contrast-enhanced ultrasound combined with three-dimensional reconstruction in preoperative perforator flap planning. Plast Reconstr Surg 2013;131(01): 80-93

24 Goh TL, Park SW, Cho JY, Choi JW, Hong JP. The search for the ideal thin skin flap: superficial circumflex iliac artery perforator flap-a review of 210 cases. Plast Reconstr Surg 2015;135(02):592-601

25 Yoshimatsu H, Iida T, Yamamoto T, Hayashi A. Superficial circumflex iliac artery-based iliac bone flap transfer for reconstruction of bony defects. J Reconstr Microsurg 2018;34(09):719-728

26 Iida T, Narushima M, Yoshimatsu H, Yamamoto T, Araki J, Koshima I. A free vascularised iliac bone flap based on superficial circumflex iliac perforators for head and neck reconstruction. J Plast Reconstr Aesthet Surg 2013;66(11):1596-1599

27 Iida T. Superficial circumflex iliac perforator (SCIP) flap: variations of the SCIP flap and their clinical applications. J Reconstr Microsurg 2014;30(07):505-508

28 Yoshimatsu H, Steinbacher J, Meng S, et al. Superficial circumflex iliac artery perforator flap: an anatomical study of the correlation of the superficial and the deep branches of the artery and evaluation of perfusion from the deep branch to the sartorius muscle and the iliac bone. Plast Reconstr Surg 2019;143(02):589-602

29 Bracaglia R, D'Ettorre M, Gentileschi S, Tambasco D. Was the surgeon a satisfactory informant? How to minimize room for claims. Aesthet Surg J 2014;34(04):632-635

30 Yoshimatsu H, YamamotoT, Iida T. Pedicle elongation technique of superficial circumflex iliac artery perforator flap. J Plast Reconstr Aesthet Surg 2015;68(03):e61-e62 